\title{
A rare neoplasm in the right atrium
}

\author{
Xingzhi Huang ${ }^{1}$, Jia $\mathrm{Hu}^{2}$, Hongling Ran ${ }^{2}$, and Juan $\mathrm{Liu}^{2}$ \\ ${ }^{1}$ The First Affiliated Hospital of Nanchang University \\ ${ }^{2}$ First Affiliated Hospital of Nanchang University
}

October 12, 2020

\begin{abstract}
Primary neoplasm of the atrium are usually thrombus or the cardiac myxomas. Cardiac hemangiomas are rare primary cardiac neoplasms. The natural evolution is uncertain due to various manifestation over time. Transthoracic echocardiography (TTE) can clearly display the location and size and clarify the impact of its relationship with myocardium, valves, and hemodynamics. We present the case of a female patient who with a complete medical history of right atrium cardiac hemangioma accidentally.
\end{abstract}

\section{KEYWORDS}

cardiac hemangioma, cardiac primary tumor, transthoracic echocardiography

A 67-year-old female patient with a 8-month history of sigmoid colon cancer resection was readmitted to our hospital due to routine follow-up. The presurgical transthoracic echocardiography (TTE) result was unremarkable (Figure 1A).

The TTE examination revealed that the right atrium (RA) had a hypoechoic neoplasm, about $4.3 \times 3.8 \mathrm{~cm}$, round, clear boundary, hyperechoic capsule, and attached to the wall (Figure 1B). The neoplasm broke into the tricuspid valve during diastole and returned to the RA during systole, leading to moderate regurgitation of the tricuspid valve during systole (Figure2; Videoclip S1). The marked hypoechogenicity and hyperechoic capsule may be the characteristic of cardiac hemangioma, which are related to thrombus and blood clots formed in the vascular lumen, and complete envelope ${ }^{1-3}$.Contrast-enhanced computerized tomography (CT) of the chest showed low-density masses with slight enhancement in the RA (Figure 3).

Surgical clipping of the atrial tumor was performed. The excised specimen was dark red in color and had a complete envelope. Histopathological examination showed tissue with benign endothelial cells and large communicating lumens consistent with a cavernous hemangioma (Figure 4). The subsequent recovery were uncomplicated and the patient was discharged 1 weeks after the operation.

\section{References}

1. Beebeejaun MY, Deshpande R. Conservative management of cardiac haemangioma. Interactive cardiovascular and thoracic surgery.2011;12(3):517-519.

2. Alpesh AP, Ebere OC, Daniel SS, et al. A right atrial hemangioma mimicking thrombus in a patient with atrial arrhythmias. The open cardiovascular medicine journal. 2007;1:34-35.

3. Xiachuan Q, Xuebin L, Yongjie W. Case of Cardiac Hemangioma Diagnosed by Myocardial Contrast Echocardiography. Circulation Cardiovascular imaging. 2019;12(5):e008811. 

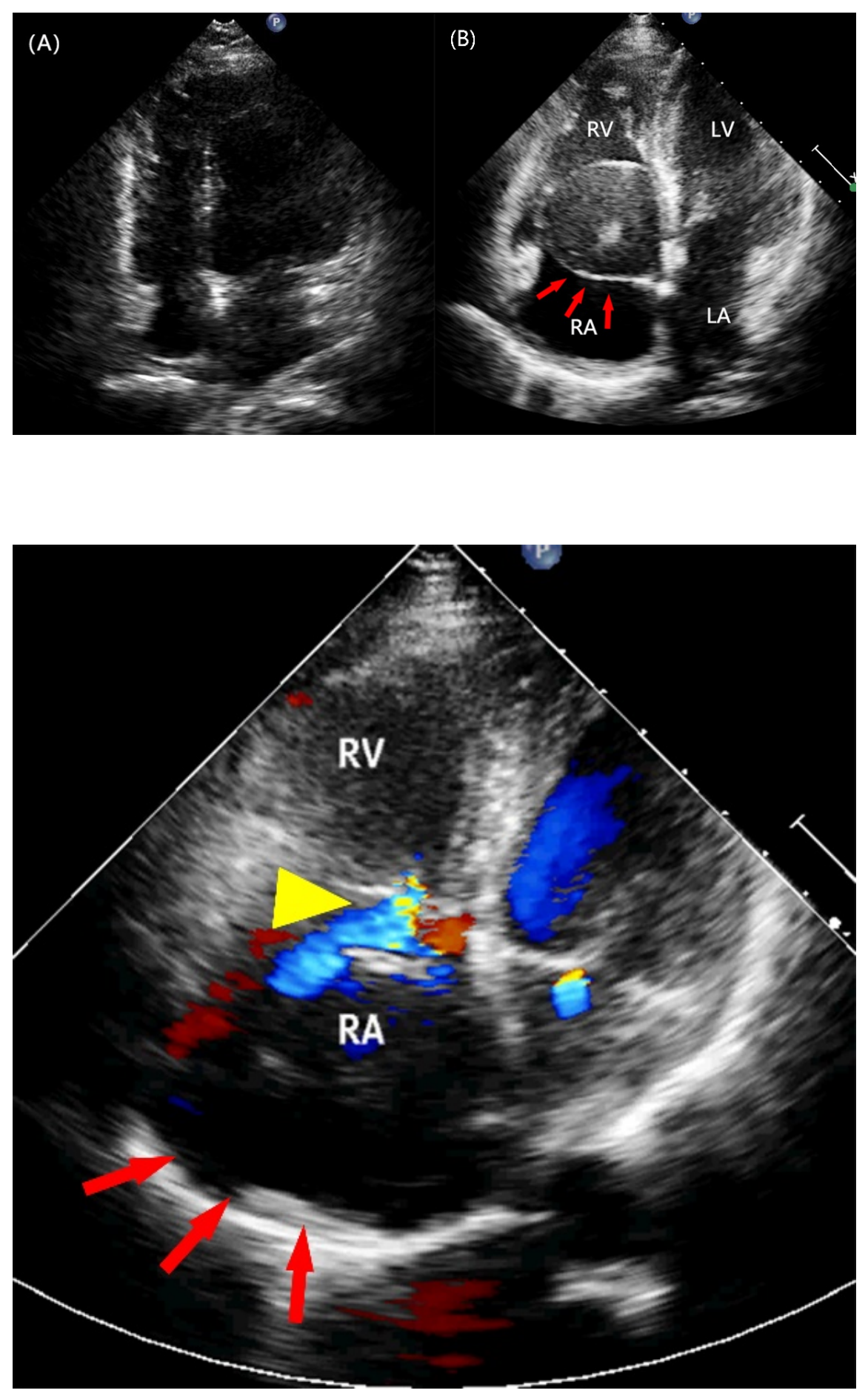


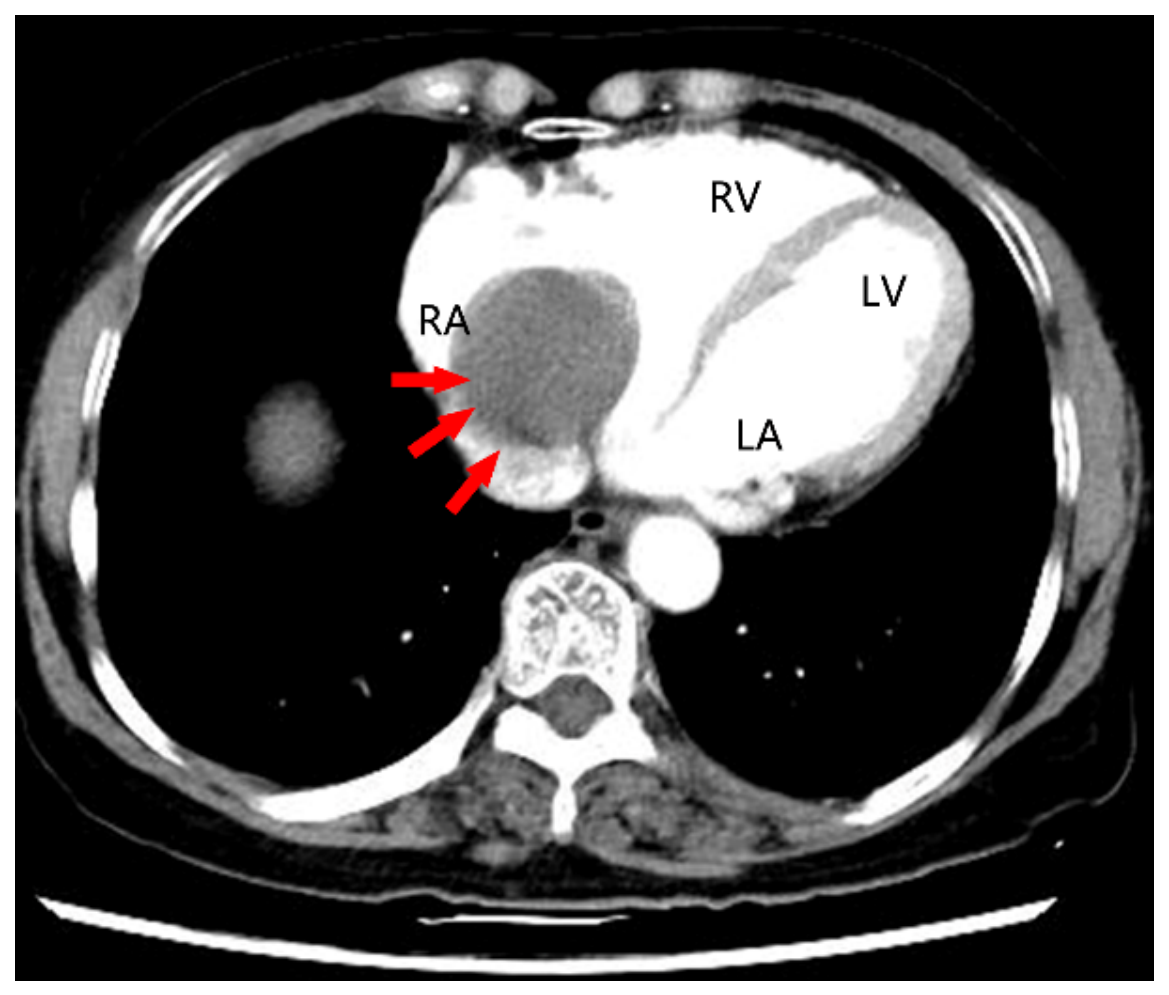




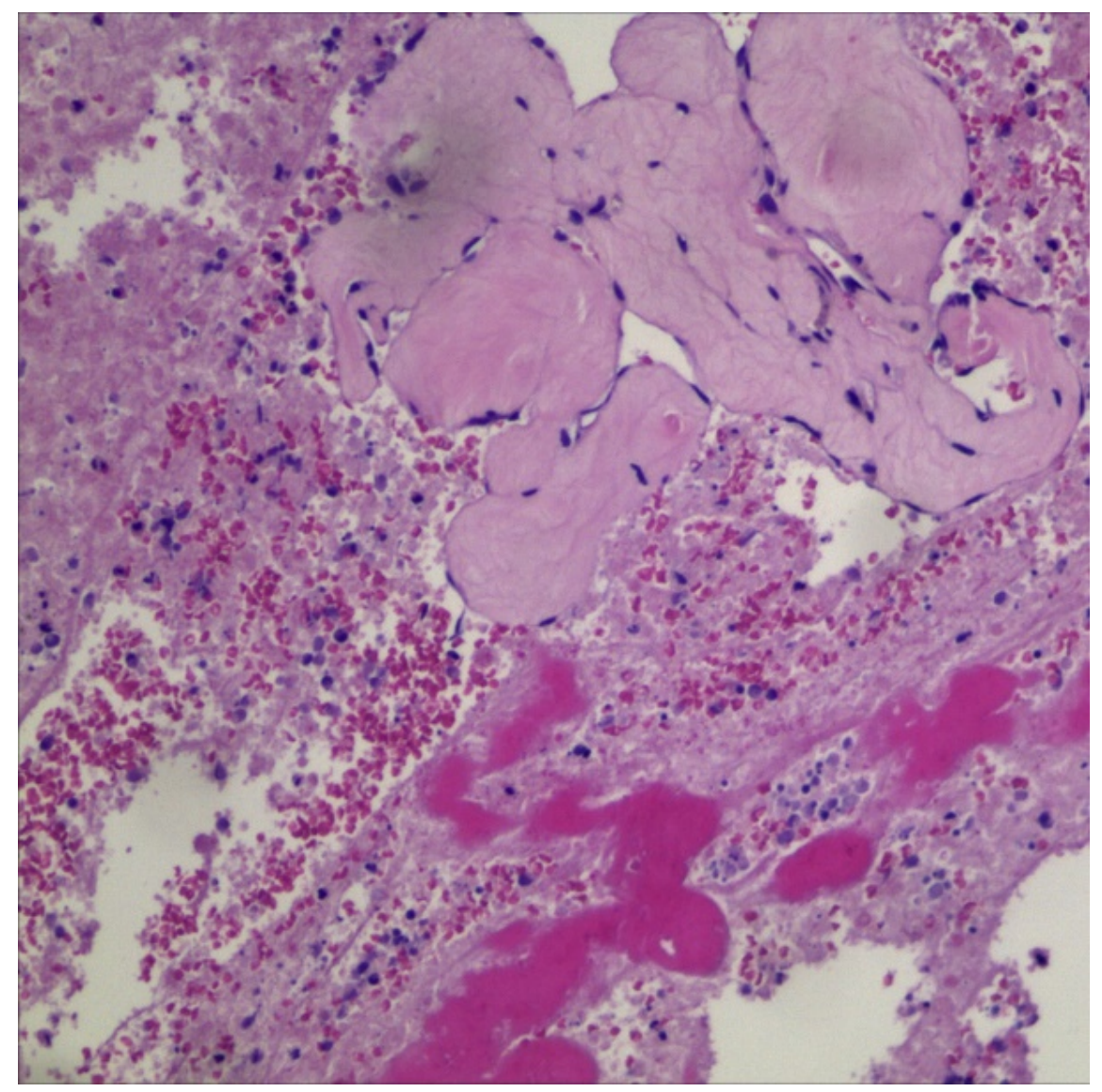

4

FIGURE 1 Transthoracic echocardiography (TTE)-modified apical 4-chambers view: A, there is unremarkable 8 months ago. B, a marked hypoechogenic neoplasm (red arrows) with a hyperechoic capsule attached to the right atrium (RA) wall, and the neoplasm breaks into the tricuspid valve during diastole.

FIGURE 2 TTE- modified parasternal short-axis view: The neoplasm (red arrows) returns to the right atrium during systole, causing moderate regurgitation of the tricuspid valve (yellow arrowhead).

FIGURE 3 Contrast-enhanced computerized tomography (CT) shows a low-density mass with little enhancement in the right atrium (red arrows)

FIGURE 4 Histopathological examination showing endothelial cells and communicating dilated lumens. Hematoxylin and eosin staining, original magnification $\times 200$. 


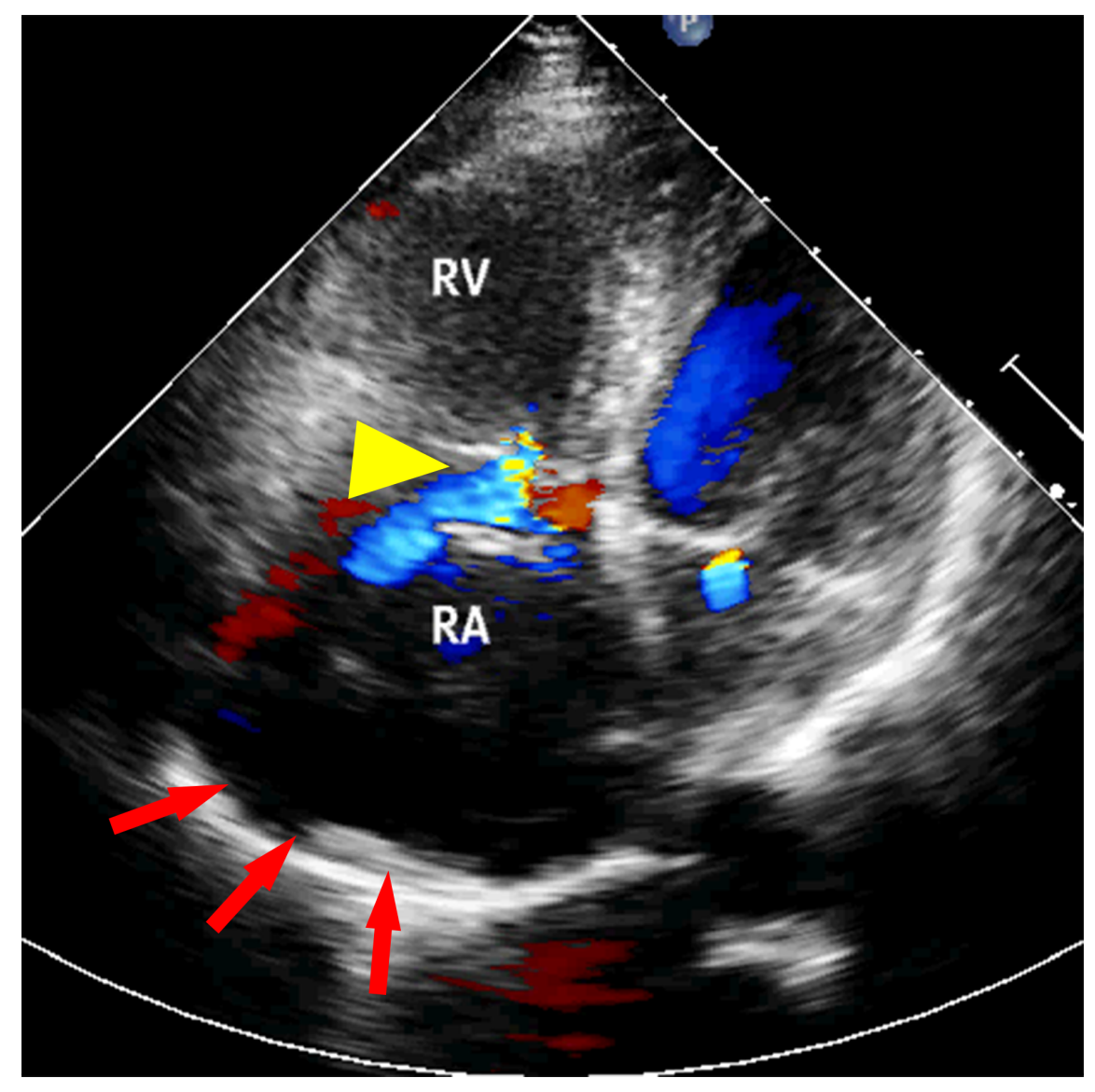



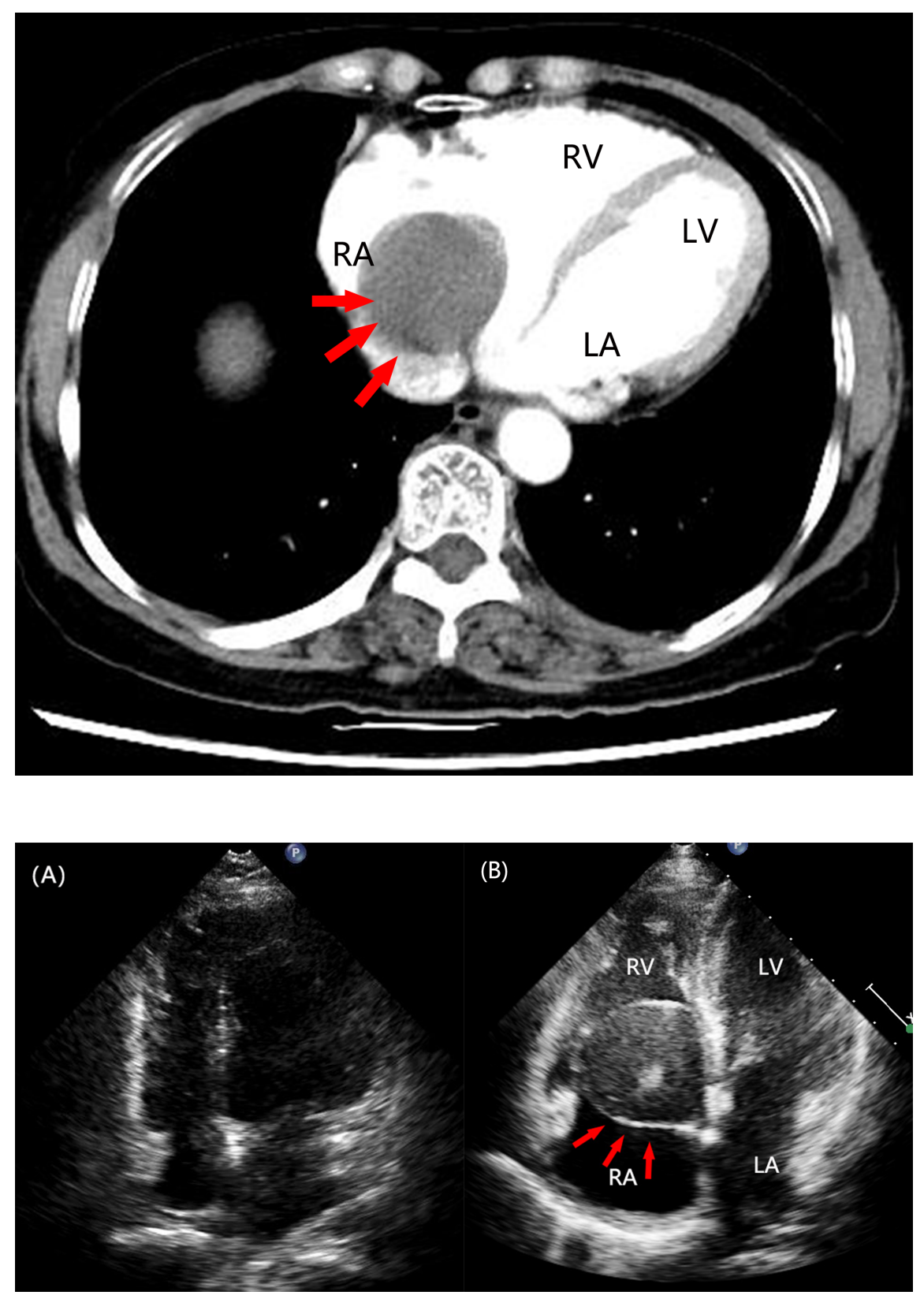


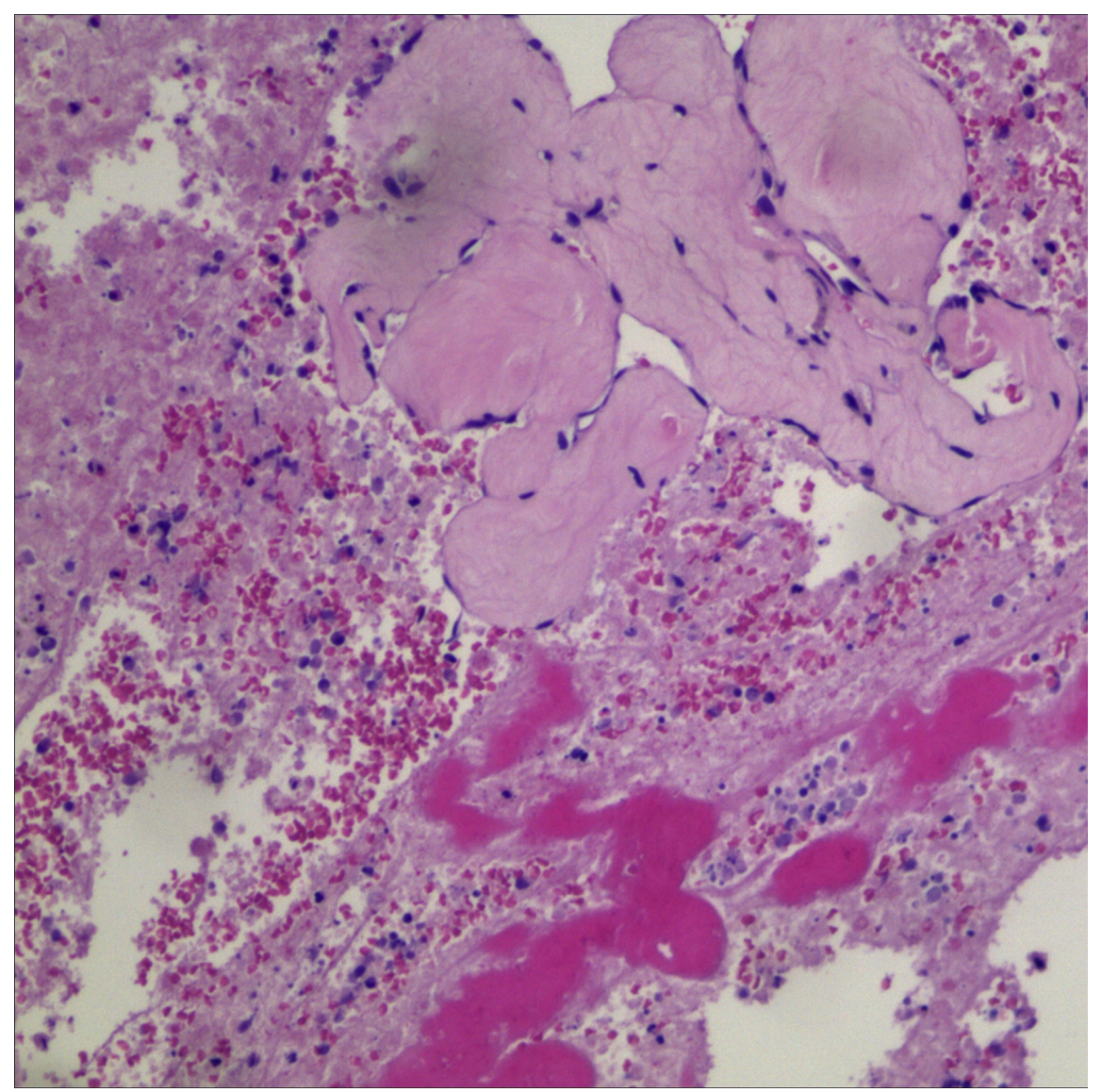

